\title{
Full Characterization of Act-and-wait Control for First-order Unstable Lag Processes
}

\section{T. INSPERGER}

Department of Applied Mechanics, Budapest University of Technology and Economics, H-1521

Budapest, Hungary (inspi@mm.bme.hu)

P. WAHI

Mechanical Engineering Department, Indian Institute of Technology - Kanpur, Kanpur, U.P., 208016, India

\section{A. COLOMBO}

Dipartimento di Elettronica e Informazione, Politecnico di Milano, Via Ponzio, 34/5-20133, Milano, Italy

\section{G. STÉPÁN}

Department of Applied Mechanics, Budapest University of Technology and Economics, H-152I Budapest, Hungary

\section{DI BERNARDO}

\section{S. J. HOGAN}

Department of Engineering Mathematics, University of Bristol, Queen's Building, University Walk, Bristol BS8 1TR, UK

(Received 30 March 2007; accepted 30 March 2008)

\begin{abstract}
Act-and-wait control is a special case of time-periodic control for systems with feedback delay, where the control gains are periodically switched on and off in order to stabilize otherwise unstable systems. The stability of feedback systems in the presence of time delay is a challenging problem. In this paper, we show that the act-and-wait type time-periodic control can always provide deadbeat control for first-order unstable lag processes with any (large but) fixed value of the time delay in the feedback loop. A full characterization of this act-and-wait controller with respect to the system and control parameters is given based on performance and robustness against disturbances.
\end{abstract}

Key words: Deadbeat, feedback delay, periodic control, stability.

\section{INTRODUCTION}

Parallel to the development of control theory, the mathematical theory of time delayed systems emerged in the early 1950s. Most of the theory of ordinary differential equations (ODEs) has been generalized for delay-differential equations (DDEs), which have infinite- 
dimensional phase spaces (Hale and Lunel, 1993; Diekmann et al., 1995). The first results for the stability analyses of linear autonomous-delayed systems appeared in the work of Hayes (1950), Bhatt and Hsu (1966) and Cushing (1977), while applications and algorithms appeared somewhat later (Kolmanovskii and Nosov,1986; Stépán, 1989; Niculescu, 2000; Olgac and Sipahi, 2002; Michiels et al., 2002; Breda et al., 2004; Butcher et al., 2004; Kalmár-Nagy, 2005; Liu et al., 2010; Mann and Patel, 2010).

The mathematical description of time-periodic nonautonomous systems goes back to the mid 19th century (Mathieu, 1868). Although a correct theoretical basis for the stability analysis of these periodic systems was provided by the Floquet theory in the 19th century (Floquet, 1883), the first stability results, such as those of Stephenson (1908) or van der Pol and Strutt (1928), appeared only much later due to the lack of a simple algorithm for stability analysis.

As both autonomous-delayed systems and time-periodic ordinary systems are quite well understood, those engineering models have received attention in recent years where the two effects, namely the delay and the time-periodic system parameter may be present together including the case of time-periodic time delay (see, for example, Namachchivaya and Beddini, 2003; Insperger and Stépán, 2004). In this respect, the most transparent engineering problem is the delayed and parametrically excited oscillator modeled by the delayed Mathieu equation (Insperger and Stépán, 2002a). Its stability analysis was motivated by several engineering applications (see, for example, Budak and Altintas, 1998).

Time delay often arises in feedback control systems where the time required for the acquisition of response and excitation data, the information transmission, or the on-line data processing is not negligible. In spite of the efforts to minimize these delays, they cannot be fully eliminated due to physical limits. The information delay may be crucial, for example, in space applications (Kim and Bejczy, 1993), in controlling congestion in the Internet (Shakkottai et al., 2003), or in robotic applications with time-consuming control force computation (Kovács et al., 2004).

Although time-invariant state feedback is a wide-spread and easily applicable technique for control systems, it does not provide a stabilizing controller for all systems. In these cases, the use of time-periodic feedback gains may improve stability properties. The problem of stabilization by means of time-periodic feedback gains in nondelayed systems has been presented by Brockett (1998) as one of the challenging open problems in control theory. Together with some papers on discrete-time systems (Aeyels and Willems, 1992; Leonov, 2002a; Artstein and Weiss, 2005; Weiss, 2005), partial results have been presented by Leonov (2002b) and Allright et al. (2005) for piecewise constant control gains and by Moreau and Aeyels (2004) for sinusoidal control gains. The solution to the problem for a wide class of systems - without delay - was recently presented by Boikov (2005).

When time-periodic gains are used in the control, stabilization means the placement of the characteristic multipliers of the system inside the unit circle of the complex plane in accordance with Floquet theory (see, for example, Farkas, 1994). In this sense, stabilization is a weak version of pole placement, where the poles are defined as the eigenvalues of the so-called Floquet transition matrix (or monodromy matrix) of the time-periodic system. The main difficulty in the above analysis is that there are no closed-form results to construct this matrix analytically.

Caused by the delay of the time-periodic control feedback, the governing equation is a nonautonomous DDE with an infinite-dimensional phase space (Hale and Lunel, 1993; 


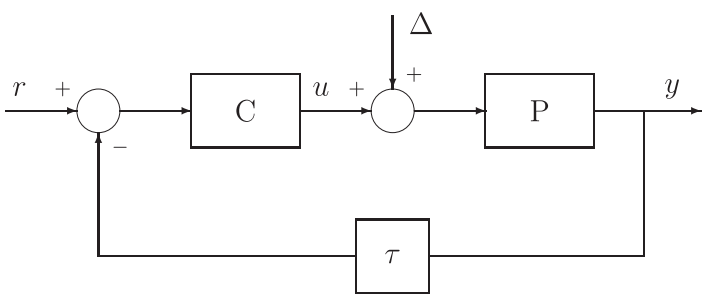

Figure 1. Block diagram of the control system with feedback delay.

Farkas, 1994). Therefore, the linear stability conditions for the system parameters are even more complicated. The infinitely many characteristic multipliers (taking the role of the system poles) are the eigenvalues of the monodromy operator (infinite-dimensional Floquet transition matrix), and these poles should be placed in the open unit disc of the complex plane to achieve stability.

Stabilization of time-delayed systems is difficult, since an infinite number of poles should be controlled using a finite number of control parameters. The act-and-wait control method is an effective technique to place infinitely many poles at the origin even for systems with large feedback delay, which makes the pole placement problem easier. It is a special case of periodic controllers in which the controller is switched on and off periodically. The method was introduced first for discrete systems by Insperger and Stépán (2007), and it was adopted for continuous-time systems by Insperger (2006) and Stépán and Insperger (2006).

In this paper, we present a full characterization of the act-and-wait control applied for the delayed first-order lag compensator. The optimization of the waiting time relative to the time delay in the feedback loop is based on the analytical construction of the monodromy operator. It is shown that deadbeat control can be achieved in a wide range of parameters if the waiting time is larger than the delay. The system performance is analyzed with respect to three different terms of the performance index including the fastest settling signal (or largest stability margin), the minimal control effort, and the minimal error for constant actuator disturbances such as Coulomb friction. The robustness of the deadbeat control strategy with respect to perturbations in the system and control parameters is also analyzed using stability radii as a measure.

The analysis gives a summary of the pros and cons of the act-and-wait control strategy that helps control engineers to choose between options such as the Smith predictor, or other well-known methods.

\section{MATHEMATICAL MODEL OF ACT-AND-WAIT CONTROL FOR A FIRST-ORDER LAG SYSTEM}

Figure 1 shows the control loop of the simplest possible system where advantages of the act-and-wait control can be discussed. The SISO plant P is a first-order system described by

$$
\begin{aligned}
& \dot{x}(t)=a x(t)+b(u(t)+\Delta), \\
& y(t)=c x(t),
\end{aligned}
$$




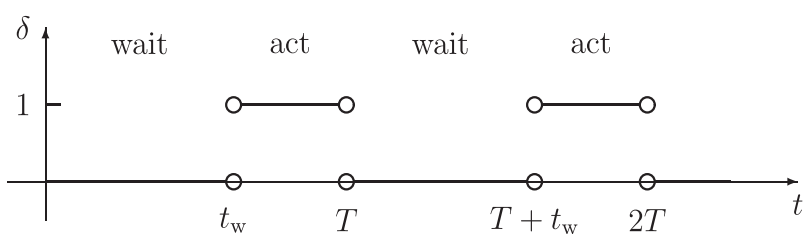

Figure 2. The act-and-wait function.

with system parameter $a>0$, which corresponds to an unstable open-loop process. The actuator disturbances are modeled by $\Delta$, which is assumed to be constant when the effect of Coulomb friction is considered with respect to the accuracy of the final position of the system in the case of stable processes.

The plant is set in a negative feedback loop that contains a feedback delay $\tau$. The controller is given by

$$
u(t)=\delta(t) d(r(t)-x(t-\tau)),
$$

where $d$ is the control gain, while

$$
\delta(t)= \begin{cases}0, & \text { if } \quad 0 \leq t \bmod T<t_{\mathrm{w}}, \\ 1, & \text { if } \quad t_{\mathrm{w}} \leq t \bmod T<t_{\mathrm{w}}+t_{\mathrm{a}}=T .\end{cases}
$$

The periodic piecewise constant function $\delta$ activates and deactivates the control. For a time period of length $t_{\mathrm{w}}$, the controller is switched off (wait), while for the next period of length $t_{\mathrm{a}}$, the controller is switched on (act), as shown in Figure 2. This is then repeated periodically with time period $T=t_{\mathrm{w}}+t_{\mathrm{a}}$ leading to the act-and-wait control strategy.

With the appropriate rescaling of time with respect to the time delay, the delay parameter (when present) can be set to $\tau=1$. Furthermore, we consider a zero reference signal: $r(t) \equiv$ 0 , and we assume, without loss of generality, that $b=1$ and $c=1$. Thus, the system under study is of the form:

$$
\begin{aligned}
& \dot{x}(t)=a x(t)+u(t)+\Delta, \\
& u(t)=-\delta(t) d x(t-1) .
\end{aligned}
$$

Since $\Delta$ is constant, it does not affect stability properties, but does affect the accuracy of the final position $x_{\mathrm{f}}$, given by

$$
x_{\mathrm{f}}=\lim _{t \rightarrow \infty} x(t)=\frac{\Delta}{d-a} .
$$




\section{MOTIVATION}

The system in equations 5 and 6 without actuator disturbances $\Delta$ gives the time-periodic DDE,

$$
\dot{x}(t)=a x(t)-\delta(t) d x(t-1) .
$$

The goal of this paper is to provide a full stability picture of equations 8 and 4 for any system parameter $a>0$ with respect to the control parameters $d, t_{\mathrm{w}}$ and $t_{\mathrm{a}}$. First, some special cases are discussed.

\subsection{Case $d=0$}

If there is no controller and no disturbance, apart from the initial condition $x(0)$ for the system, we are left with

$$
\dot{x}(t)=a x(t) \quad \Rightarrow \quad x(t)=\mathrm{e}^{a t} x(0),
$$

leading to $x_{\mathrm{f}} \rightarrow \pm \infty$ for $a>0$.

\subsection{Case $d \neq 0, \delta(t) \equiv 1, \tau=0$}

If there is no time delay in the feedback loop, then constant control gains can also be used to stabilize the system. In this case, the governing equation and the system response become

$$
\dot{x}(t)=(a-d) x(t) \Rightarrow x(t)=\mathrm{e}^{(a-d) t} x(0) .
$$

Therefore, a control gain $d>a$ always provides a stable process.

\subsection{Case $d \neq 0, \delta(t) \equiv 1, \tau=1$}

In the presence of time delay, constant gains can still produce a stable process. The corresponding equation becomes

$$
\dot{x}(t)=a x(t)-d x(t-1)
$$

Due to the time delay, this system has an infinite number of characteristic roots. Stability properties of this system are described by the so called Hayes diagram (Hayes, 1950) shown in Figure 3. This equation is often considered to be one of the simplest basic examples for a delayed system (see, for example, Stépán, 1989; Michiels et al., 2002). The stability boundaries (thick lines in Figure 3) and the parameters corresponding to largest stability margin (dashed line in Figure 3) can be determined through the analysis of the characteristic equation. If $a>1$, then this system is always unstable for any value of $d$. 


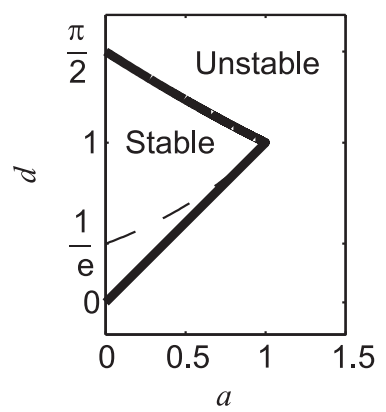

Figure 3. The Hayes diagram for equation 11.

\subsection{The act-and-wait control strategy}

The equation under investigation now becomes

$$
\dot{x}(t)=a x(t)-\delta(t) d x(t-1),
$$

where $\delta(t)$ is given by equation 4 . The general solution of this DDE for the initial function $x_{0}$ can be formulated as

$$
x_{t}=\mathcal{U}(t) x_{0}
$$

where $\mathcal{U}(t)$ is the solution operator of the system, the function $x_{t}$ is defined by the shift

$$
x_{t}(s)=x(t+s), \quad s \in[-1,0],
$$

and $x_{0}(s)$ is the initial function for the system. Stability properties are determined by the monodromy operator $\mathcal{U}(T)$. The nonzero elements of the spectrum of $\mathcal{U}(T)$ are called characteristic multipliers (or poles), also defined by

$$
\operatorname{Ker}(\mu \mathcal{I}-\mathcal{U}(T)) \neq\{0\}
$$

The system is asymptotically stable if all the characteristic multipliers lie in the open unit disc of the complex plane. Since DDEs usually have infinitely many poles, stability properties cannot be given in closed form, but numerical techniques can be used to construct stability charts.

The point of the act-and-wait concept is that, if the waiting period is longer than the time delay, then the monodromy operator becomes finite-dimensional and can be represented as a monodromy matrix of the same dimension as the plant (see, Insperger, 2006). In our case, this means that, if $t_{\mathrm{w}} \geq 1$, then equation 12 can be transformed into a scalar discrete map. This does not hold for the case $t_{\mathrm{w}}<1$.

Figure 4 shows a series of stability charts for equation 12 in the parameter plane $(a, d)$ for different waiting and acting period lengths. The charts were determined numerically by 

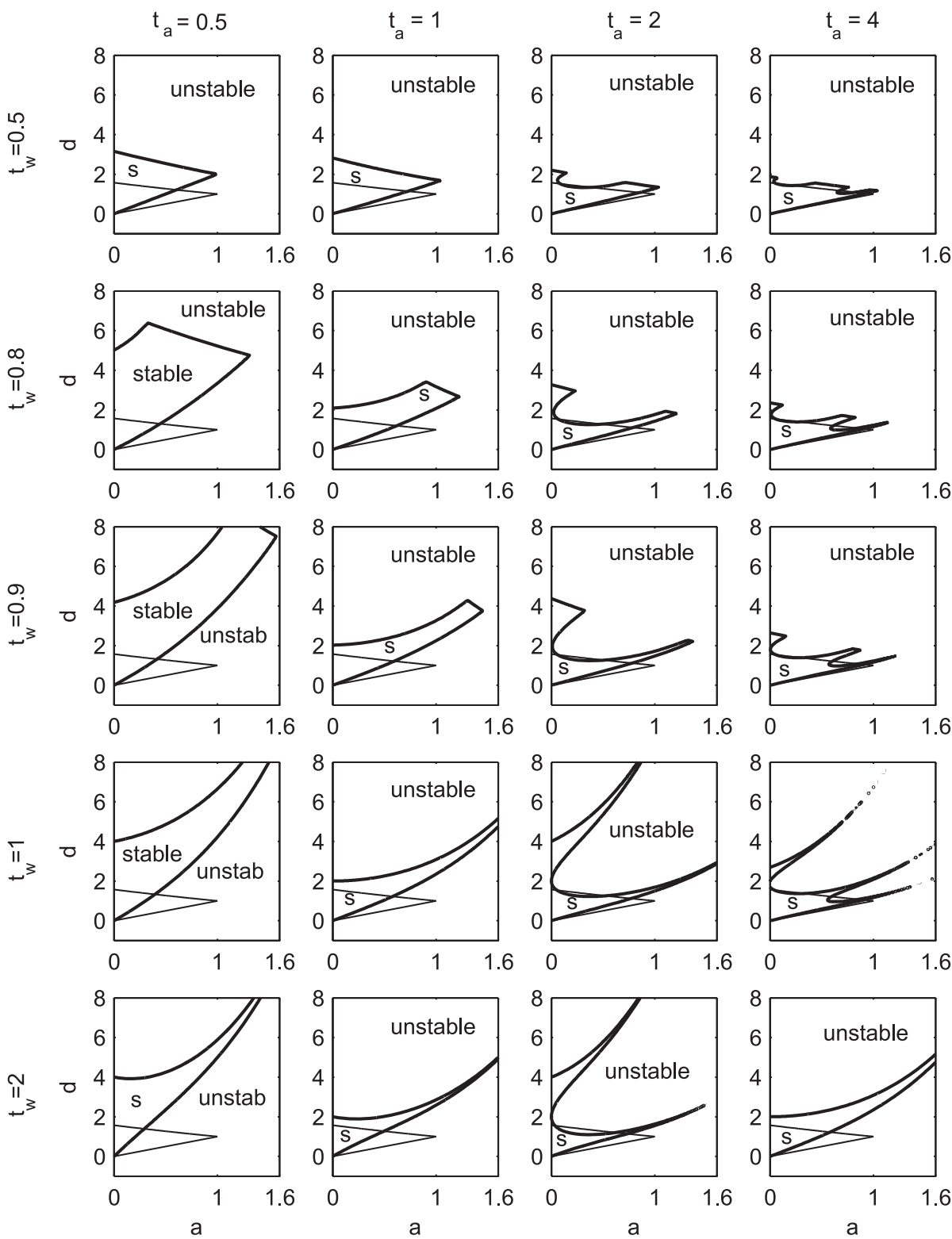

Figure 4. Stability charts for equation 12 . Thick lines: stability boundaries for the act-and-wait control system. Thin lines: stability boundaries for the case $\delta(t) \equiv 1$.

computing the critical (maximum in modulus) poles over a $200 \times 200$ sized grid of the parameters $a$ and $d$ using the semi-discretization method (Insperger and Stépán, 2002b). For comparison, the stability boundaries corresponding to the Hayes diagram (when $\delta(t) \equiv 1$ ) are also shown by thin lines. It can be seen that the stable domains are essentially larger (for certain waiting and acting period lengths) than those of the Hayes diagram. 
The case with $t_{\mathrm{w}}=1$ and $t_{\mathrm{a}}=1$ has already been investigated in Stépan and Insperger (2006) as a case study. It was shown that the system is described by a scalar discrete map, and for any system parameter $a$, deadbeat control can be achieved. Here, this system will be investigated for a wider range of the parameters $a, d, t_{\mathrm{w}}$ and $t_{\mathrm{a}}$, including the case when $t_{\mathrm{w}}<1$.

\section{OPTIMIZATION OF WAITING TIME}

Stability properties of equation 12 are determined by the monodromy operator $\mathcal{U}(T)$ that maps the initial function $x_{0}$ into $x_{T}$. This means that the state $x(T)$ generally depends on the initial function $x_{0}(s)$, and it cannot be determined using a single initial value, say $x(0)$. However, in some special cases of the act-and-wait control system, finite-dimensional discrete maps can be constructed between finitely many states of the initial function $x_{0}$ and the function $x_{T}$ after time $T$. In these cases, the delayed systems become finite-dimensional, and the stability properties are also determined by a finite number of poles. These cases will be investigated in the following subsections.

\subsection{Case $t_{\mathrm{w}} \geq 1$}

In this case, a closed-form solution can be determined for any acting period $t_{\mathrm{a}}$. Assume, the general case $n-1<t_{\mathrm{a}} \leq n$, where $n$ is an arbitrary positive integer. Since the delayed term is switched off during the waiting period, the first section of the solution can be given as

$$
x_{\mathrm{w}}(t)=\mathrm{e}^{a t} x(0), \quad 0 \leq t \leq t_{\mathrm{w}} .
$$

Here, subscript $w$ refers to the solution over the waiting period.

Now, we use the fact that the waiting period is larger than (or equal to) the time delay, and that the solution over $0 \leq t \leq t_{\mathrm{w}}$ is given by equation 16 . Thus, in the interval $t_{\mathrm{w}}<t \leq t_{\mathrm{w}}+1$, equation 12 can be written as

$$
\dot{x}(t)=a x(t)-d \mathrm{e}^{a(t-1)} x(0), \quad t_{\mathrm{w}}<t \leq t_{\mathrm{w}}+1,
$$

and its solution for the initial condition $x\left(t_{\mathrm{w}}\right)=\mathrm{e}^{a t_{\mathrm{w}}} x(0)$ is given by

$$
x_{\mathrm{a} 1}(t)=\underbrace{\mathrm{e}^{a t} x(0)}_{=x_{\mathrm{w}}(t)}-d \mathrm{e}^{a(t-1)} x(0) \int_{t_{\mathrm{w}}}^{t} s, \quad t_{\mathrm{w}}<t \leq t_{\mathrm{w}}+1 .
$$

Here, subscript a1 refers to the solution over the first section of the acting period.

For $t_{\mathrm{w}}+1<t \leq t_{\mathrm{w}}+2$, equation 12 becomes

$$
\dot{x}(t)=a x(t)-d\left(\mathrm{e}^{a(t-1)} x(0)-d \mathrm{e}^{a(t-2)} x(0) \int_{t_{\mathrm{w}}}^{t-1} \mathrm{~d} s\right), \quad t_{\mathrm{w}}+1<t \leq t_{\mathrm{w}}+2,
$$

and its solution for the initial condition $x\left(t_{\mathrm{w}}+1\right)=x_{\mathrm{a} 1}\left(t_{\mathrm{w}}+1\right)$ is given by 


$$
\begin{aligned}
& x_{\mathrm{a} 2}(t)=\underbrace{\mathrm{e}^{a t} x(0)-d \mathrm{e}^{a(t-1)} x(0) \int_{t_{\mathrm{w}}}^{t} \mathrm{~d} s}_{=x_{\mathrm{a} 1}(t)}+d^{2} \mathrm{e}^{a(t-2)} x(0) \int_{t_{\mathrm{w}}+1}^{t} \int_{t_{\mathrm{w}}}^{s_{2}-1} \mathrm{~d} s_{1} \mathrm{~d} s_{2}, \\
& t_{\mathrm{w}}+1<t \leq t_{\mathrm{w}}+2 .
\end{aligned}
$$

Generally, it can be shown that the solution over the interval $t_{\mathrm{w}}+k-1<t \leq t_{\mathrm{w}}+k$ is given by

$$
x_{\mathrm{a} k}(t)=x_{\mathrm{a} k-1}+(-1)^{k} d^{k} \mathrm{e}^{a(t-k)} I_{k}(t) x(0),
$$

where $I_{k}(t)$ is a nested integral defined recursively as

$$
I_{k}(t)=\int_{t_{\mathrm{w}}+k-1}^{t} I_{k-1}\left(s_{k}-1\right) \mathrm{d} s_{k},
$$

with $I_{0}(t) \equiv 1$, which can be evaluated in closed form as

$$
I_{k}(t)=\frac{\left(t-t_{\mathrm{w}}-k+1\right)^{k}}{k !} .
$$

Thus, the solution in the interval $t_{\mathrm{w}}+n-1<t \leq t_{\mathrm{w}}+n$ can be written in the compact form

$$
x_{\mathrm{a} n}(t)=\mathrm{e}^{a t}\left(\sum_{k=0}^{n} \frac{\left(-d \mathrm{e}^{-a}\right)^{k}\left(t-t_{\mathrm{w}}-k+1\right)^{k}}{k !}\right) x(0) .
$$

Substitution of $t=t_{\mathrm{a}}+t_{\mathrm{w}}=T$ into equation 24 yields the discrete map $\Phi_{n}$ from $x(0)$ to $x(T)$ as

$$
\Phi_{n}=\mathrm{e}^{a\left(t_{\mathrm{a}}+t_{\mathrm{w}}\right)}\left(\sum_{k=0}^{n} \frac{\left(-d \mathrm{e}^{-a}\right)^{k}\left(t_{\mathrm{a}}-k+1\right)^{k}}{k !}\right) .
$$

This way, the monodromy mapping

$$
x_{T}=\mathcal{U}(T) x_{0},
$$

is reduced to

$$
\left(\begin{array}{c}
x(T) \\
\tilde{x}_{T}
\end{array}\right)=\left(\begin{array}{cc}
\Phi_{n} & O \\
f_{n} & \mathcal{O}
\end{array}\right)\left(\begin{array}{c}
x(0) \\
\tilde{x}_{0}
\end{array}\right),
$$

where function $\tilde{x}_{t}$ is defined by the shift

$$
\tilde{x}_{t}(s)=x(t+s), \quad s \in[-1,0) .
$$


Note that $s=0$ is excluded here as opposed to equation 14. In equation 27, $O$ denotes the zero functional, $\mathcal{O}$ denotes the zero operator and $f_{n}$ is a function given from the solution in equation 24 as

$$
f_{n}(s)= \begin{cases}\mathrm{e}^{a s}\left(\sum_{k=0}^{n-1} \frac{\left(-d \mathrm{e}^{-a}\right)^{k}\left(s+T-t_{\mathrm{w}}-k+1\right)^{k}}{k !}\right), & \text { if } \quad-1 \leq s<n-1-t_{\mathrm{a}}, \\ \mathrm{e}^{a s}\left(\sum_{k=0}^{n} \frac{\left(-d \mathrm{e}^{-a}\right)^{k}\left(s+T-t_{\mathrm{w}}-k+1\right)^{k}}{k !}\right), & \text { if } \quad n-1-t_{\mathrm{a}} \leq s<0 .\end{cases}
$$

Equation 27 shows that function $x_{T}$ can be determined using only the initial value $x(0)$ and does not depend on the initial function $\tilde{x}_{0}$. Thus, the monodromy operator has only 1 nonzero eigenvalue that is just equal to $\Phi_{n}$, and all the further infinitely many eigenvalues are set to zero. Clearly, the system is stable if $\left|\Phi_{n}\right| \leq 1$.

For $0<t_{\mathrm{a}} \leq 1$, equation 25 gives

$$
\Phi_{1}=\mathrm{e}^{a\left(t_{\mathrm{a}}+t_{\mathrm{w}}\right)}\left(1-d \mathrm{e}^{-a} t_{\mathrm{a}}\right) .
$$

If $1<t_{\mathrm{a}} \leq 2$, then

$$
\Phi_{2}=\mathrm{e}^{a\left(t_{\mathrm{a}}+t_{\mathrm{w}}\right)}\left(1-d \mathrm{e}^{-a} t_{\mathrm{a}}+\frac{1}{2} d^{2} \mathrm{e}^{-2 a}\left(t_{\mathrm{a}}-1\right)^{2}\right) .
$$

Figure 5 shows a stability chart in the parameter plane $\left(d, t_{\mathrm{a}}\right)$. The stability boundaries in the domains $n-1<t_{\mathrm{a}} \leq n, n=1,2,3,4$ were determined by solving $\Phi_{n}= \pm 1$ for $\delta$ and $t_{\mathrm{a}}$ using equation 25 .

The condition for deadbeat control is $\Phi_{n}=0$. Using equation 25 , this gives

$$
\sum_{k=0}^{n} \frac{(-1)^{k}\left(t_{\mathrm{a}}-k+1\right)^{k} \mathrm{e}^{-k a}}{k !} d^{k}=0 .
$$

Note that this condition depends only on the parameter triples $a, t_{\mathrm{a}}$ and $d$, and does not depend on the waiting period $t_{\mathrm{w}}$. For a given system parameter $a$ and acting period $n-1<$ $t_{\mathrm{a}} \leq n$, the left-hand side of 32 is a polynomial in $d$ of order $n$. Hence, in the case of real polynomial roots, there are $n$ distinct values of $d$ for deadbeat control. For $0<t_{\mathrm{a}} \leq 1$, deadbeat control is achieved if

$$
d=\mathrm{e}^{a} / t_{\mathrm{a}}
$$

For $1<t_{\mathrm{a}} \leq 2, \Phi_{2}=0$ gives

$$
d_{1,2}=\frac{t_{\mathrm{a}} \pm \sqrt{-t_{\mathrm{a}}^{2}+4 t_{\mathrm{a}}-2}}{\left(t_{\mathrm{a}}-1\right)^{2}} \mathrm{e}^{a} .
$$

For a given $1<t_{\mathrm{a}} \leq 2$, there exist two values of $d$ that result in deadbeat control. For larger $t_{\mathrm{a}}, \Phi_{3}=0, \Phi_{4}=0$, etc. should be considered, and further deadbeat parameter curves appear, as shown in Figure 5 by dashed lines. 


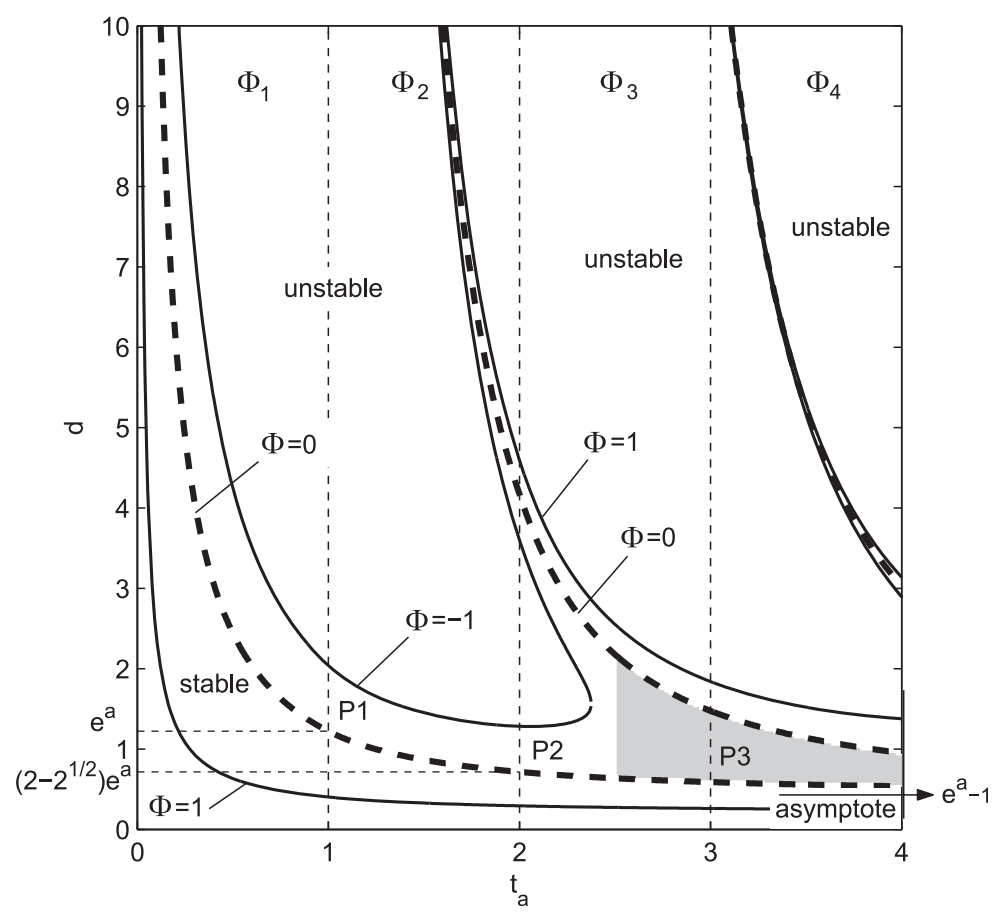

Figure 5. Stability chart for equation 12 with $a=0.2$ and $t_{\mathrm{w}}=1$. Solid lines: stability boundaries for the act-and-wait control system. Dashed lines: parameter curves for deadbeat control.

The values of the control gain parameter $d$ at the intersection points of the lowest deadbeat parameter curve and the vertical lines $t_{\mathrm{a}}=1,2, \ldots$ can be computed using 32 . The first section point (P1 in Figure 5) corresponds to $d_{\mathrm{P} 1}=\mathrm{e}^{a}$, the second point (P2 in Figure 5) corresponds to $d_{\mathrm{P} 2}=(2-\sqrt{2}) \mathrm{e}^{a}$. For $t_{\mathrm{a}} \rightarrow \infty$, numerical computation gives the conjecture

$$
\lim _{n \rightarrow \infty} d_{\mathrm{P} n}=\mathrm{e}^{a-1} .
$$

Thus, the line $d=\mathrm{e}^{a-1}$ is an asymptote of the deadbeat curve. This means that deadbeat control cannot be achieved if $d<\mathrm{e}^{a-1}$ (due to some restriction on the maximal control gain).

In Figure 5, the gray color denotes an interesting parameter domain. This gray area is surrounded by deadbeat parameter curves with no stability boundaries in between them. This means that the stability properties of the corresponding system is very robust with respect to perturbations in $d$. If $t_{\mathrm{a}}$ is fixed and $d$ is perturbed, then the system moves to the direction of deadbeat for both decreasing and increasing $d$ without loosing stability.

Since $\Phi_{n}$ is proportional to $\mathrm{e}^{a t_{\mathrm{w}}}$, its magnitude increases with increasing $t_{\mathrm{w}}$. This means that the stable domains of the stability charts shrinks with increasing $t_{\mathrm{w}}$. On the other hand, the reduction of the infinite-dimensional problem to the scalar problem described by equation 25 is possible only if $t_{\mathrm{w}} \geq 1$. Consequently, the optimal choice of the waiting period is $t_{\mathrm{w}}=1$. 

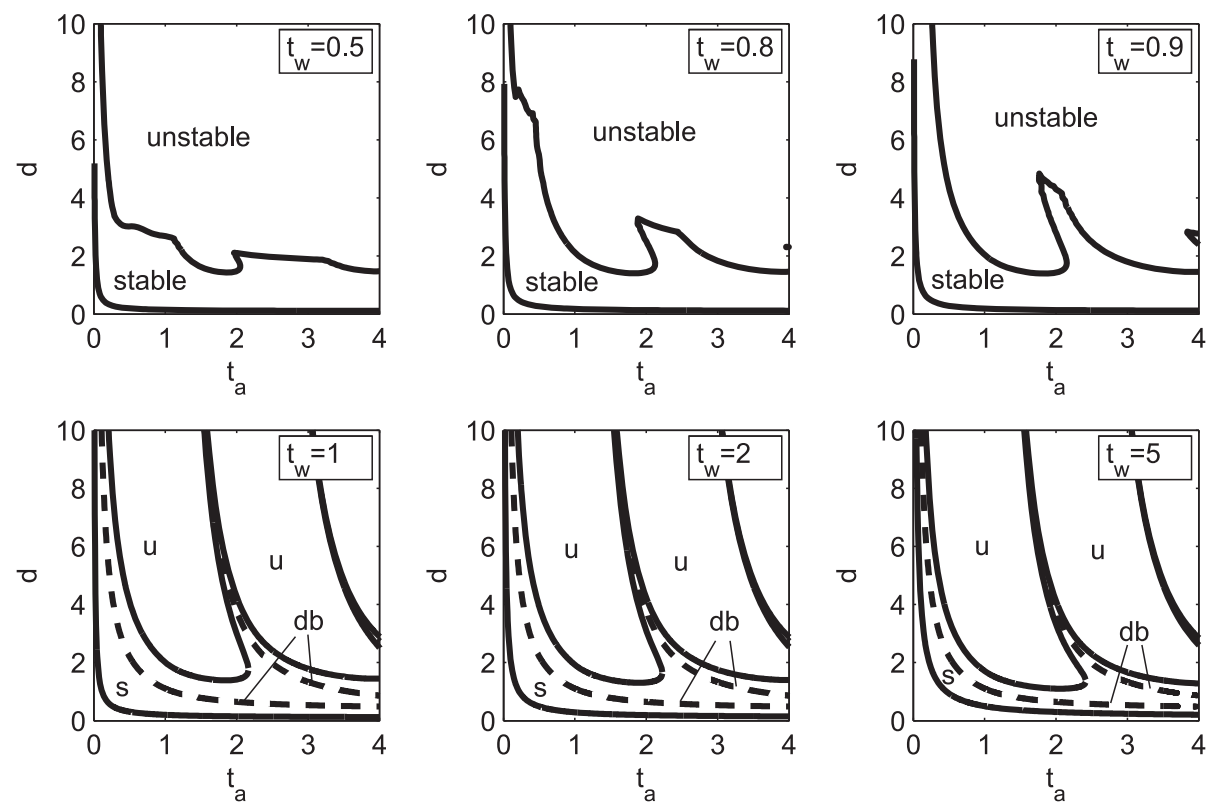

Figure 6. Stability chart for equation 12 with $a=0.1$. Continuous lines: stability boundaries. Dashed lines: deadbeat control.

However, the choice of $t_{\mathrm{w}}=1$ may produce some sensitivity problems, since some small perturbations either in $t_{\mathrm{w}}$ or in the delay $\tau=1$ may result in $t_{\mathrm{w}}<\tau$. In that case, the scalar map $\Phi_{n}$ cannot be constructed, and the system can suddenly become infinitedimensional. In order to investigate this issue, some stability charts are presented in Figure 6 for different values of $t_{\mathrm{w}}$. The charts for $t_{\mathrm{w}}=0.5,0.8$ and 0.9 were constructed using the semi-discretization method (Insperger and Stépán, 2002b). For the cases $t_{\mathrm{w}}=1,2,5$, the charts were determined by solving $\Phi_{n}= \pm 1$ for fixed values of $t_{\mathrm{a}}$.

For $t_{\mathrm{w}}>1$, it can be seen that the stable domains shrink slightly for increasing $t_{\mathrm{w}}$. The numerical plots for $t_{\mathrm{w}}<1$ show that the transition between the cases $t_{\mathrm{w}}<1$ and $t_{\mathrm{w}}>1$ is smooth, no abrupt change in the stability boundaries can be detected. However, one significant difference is that, while deadbeat control is possible for $t_{\mathrm{w}} \geq 1$, it is not possible for $t_{\mathrm{w}}<1$, as will be shown in the next subsection.

\subsection{Case $t_{\mathrm{w}}<1$}

The constructive step-by-step solution presented in the previous subsection is not valid if the waiting period is shorter than the time delay $\left(t_{\mathrm{w}}<1\right)$. It can be shown that, under certain conditions, the system can still be transformed into a finite-dimensional map. Consider the following cases:

(a) case $t_{\mathrm{w}}<1$ with $n+1-t_{\mathrm{w}}<T \leq n+t_{\mathrm{w}}$, see Figure 7a;

(b) case $t_{\mathrm{w}}<1$ with $n<T \leq n+1-t_{\mathrm{w}}$, see Figure 7b;

(c) case $t_{\mathrm{w}}<1$ with $n-1+t_{\mathrm{w}}<T \leq n$, see Figure 7c. 
(a)

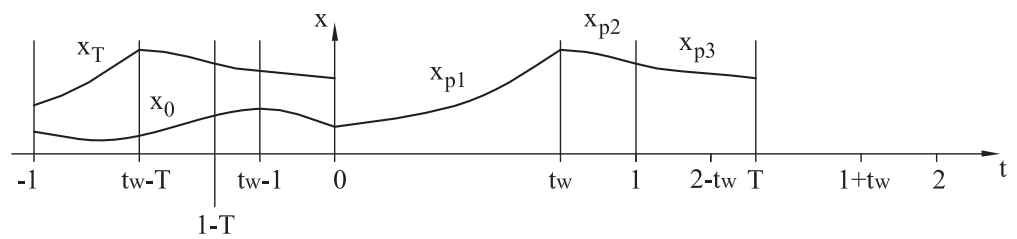

(b)

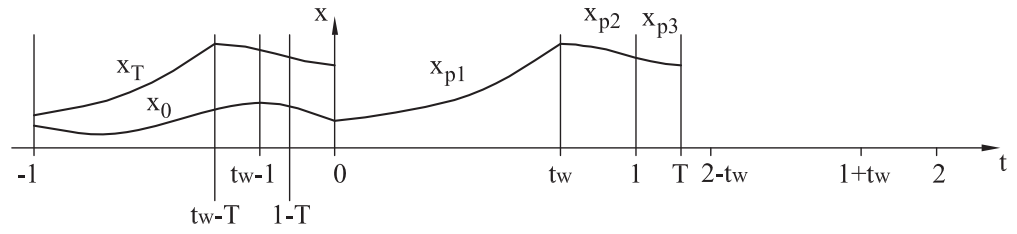

(c)

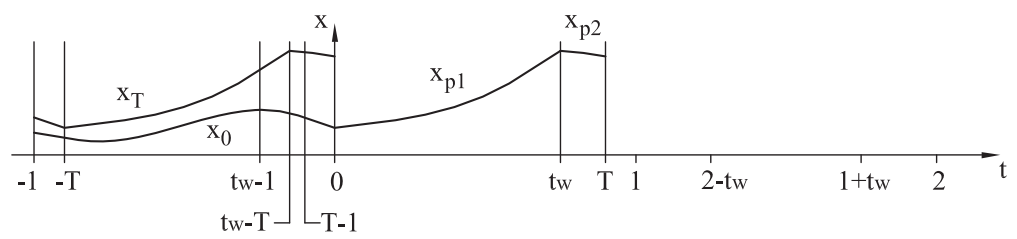

Figure 7. Graphs of the solution of equation 12 for different cases: (a) $t_{\mathrm{w}}<1, n+1-t_{\mathrm{w}}<T \leq n+t_{\mathrm{w}}$; (b) $t_{\mathrm{w}}<1, n<T \leq n+1-t_{\mathrm{w}}$; and (c) $t_{\mathrm{w}}<1, n-1+t_{\mathrm{w}}<T \leq n$.

4.2.1. Case $t_{\mathrm{w}}<1$, with $n+1-t_{\mathrm{w}}<T \leq n+t_{\mathrm{w}}$

Consider first the case $n=1$, i.e. $2-t_{\mathrm{w}}<T \leq 1+t_{\mathrm{w}}$. The solution over one act-andwait period is divided into three parts $\left[0, t_{\mathrm{w}}\right],\left[t_{\mathrm{w}}, 1\right]$ and $[1, T]$, as shown in Figure $7 \mathrm{a}$. For $0<t \leq t_{\mathrm{w}}$, the solution is

$$
x_{\mathrm{p} 1}(t)=\mathrm{e}^{a t} x(0)
$$

where the subscript $\mathrm{p} 1$ refers to part 1 . Integration gives the second part for $t_{\mathrm{w}}<t \leq 1$,

$$
x_{\mathrm{p} 2}(t)=\mathrm{e}^{a t} x(0)-d \int_{t_{\mathrm{w}}-1}^{t-1} \mathrm{e}^{a(t-s-1)} x_{0}(s) \mathrm{d} s .
$$

The state $x(1)$ is given by

$$
x(1)=\mathrm{e}^{a} x(0)-d \underbrace{\int_{t_{\mathrm{w}}-1}^{0} \mathrm{e}^{-a s} x_{0}(s) \mathrm{d} s}_{=: Y_{1,0}} .
$$

Here, $Y_{1,0}$ is a special weighted integral of the initial function $x_{0}$. Finally, the third part of the solution for $1<t \leq T$ is given by 


$$
x_{\mathrm{p} 3}(t)=\mathrm{e}^{a t}\left(1-d \mathrm{e}^{-a}(t-1)\right) x(0)-d \mathrm{e}^{a(t-1)} Y_{1,0} .
$$

Thus, the state after one act-and-wait period is

$$
x(T)=\mathrm{e}^{a T}\left(1-d \mathrm{e}^{-a}(T-1)\right) x(0)-d \mathrm{e}^{a(T-1)} Y_{1,0} .
$$

Here, $x(T)$ is determined as a linear combination of the initial state $x(0)$ and the weighted integral $Y_{1,0}$. In order to obtain a discrete map, the value of

$$
Y_{1, T}:=\int_{t_{\mathrm{w}}-1}^{0} \mathrm{e}^{-a s} x_{T}(s) \mathrm{d} s
$$

should also be expressed as a linear combination of $x(0)$ and $Y_{1,0}$. Here, $x_{T}$ can be expressed as

$$
x_{T}(s)=\left\{\begin{array}{ccc}
x_{\mathrm{p} 1}(s+T), & \text { if } & -1 \leq s \leq t_{\mathrm{w}}-T, \\
x_{\mathrm{p} 2}(s+T), & \text { if } & t_{\mathrm{w}}-T \leq s \leq 1-T, \\
x_{\mathrm{p} 3}(s+T), & \text { if } & 1-T \leq s \leq 0 .
\end{array}\right.
$$

Thus, integration of equation 41 gives

$$
Y_{1, T}=\int_{t_{\mathrm{w}}-1}^{0} \mathrm{e}^{-a s} x_{\mathrm{p} 3}(s+T) \mathrm{d} s .
$$

Note that the solution segment $x_{\mathrm{p} 3}$ is given in equation 39. After evaluation of the integrals, it can be seen that $Y_{1, T}$ can be given as a linear combination of $x(0)$ and $Y_{1,0}$. This implies the discrete map

$$
\left(\begin{array}{c}
x(T) \\
Y_{1, T}
\end{array}\right)=\Psi_{1}\left(\begin{array}{c}
x(0) \\
Y_{1,0}
\end{array}\right)
$$

where $\Psi_{1}$ is a $2 \times 2$ matrix,

$$
\Psi_{1}=\left(\begin{array}{cc}
\mathrm{e}^{a T}\left(1-d \mathrm{e}^{-a}(T-1)\right) & -d \mathrm{e}^{a(T-1)} \\
\mathrm{e}^{a(T-1)}\left(1-t_{\mathrm{w}}\right)\left(\mathrm{e}^{a}+\frac{1}{2} d\left(t_{\mathrm{w}}+2 T-3\right)\right) & -d \mathrm{e}^{a(T-1)}\left(1-t_{\mathrm{w}}\right)
\end{array}\right)
$$

This way, the monodromy mapping can be written in the form

$$
\left(\begin{array}{c}
x(T) \\
Y_{1, T} \\
w_{T}
\end{array}\right)=\left(\begin{array}{ccc}
\Psi_{1(11)} & \Psi_{1(12)} & O \\
\Psi_{1(21)} & \Psi_{1(22)} & O \\
f_{x} & f_{Y 1} & \mathcal{O}
\end{array}\right)\left(\begin{array}{c}
x(0) \\
Y_{1,0} \\
w_{0}
\end{array}\right)
$$

Here, function $w_{t}$ is defined as 


$$
w_{t}(s)=x_{t}-x_{t}(0) s_{x}-Y_{1, t} s_{Y 1},
$$

where

$$
Y_{1, t}=\int_{t_{\mathrm{w}}-1}^{0} \mathrm{e}^{-a s} x_{\mathrm{p} 3}(s+t) \mathrm{d} s,
$$

and $s_{x}$ and $s_{Y 1}$ are the right eigenvectors of the monodromy operator $\mathcal{U}(T)$ corresponding to $x_{t}(0)$ and $Y_{1, t}$, respectively. Functions $f_{x}$ and $f_{Y 1}$ describe the dependence of $w_{T}$ on $x(0)$ and $Y_{1,0}$. Equation 46 shows that the system can be reduced to a $2 \times 2$ system governed by $\Psi_{1}$. All the remaining infinitely many eigenvalues are set to zero.

Consider now the case $n=2$, i.e. $3-t_{\mathrm{w}}<T \leq 2+t_{\mathrm{w}}$. In this case, a three-dimensional discrete map can be constructed:

$$
\left(\begin{array}{c}
x(T) \\
Y_{1, T} \\
Y_{2, T}
\end{array}\right)=\Psi_{2}\left(\begin{array}{c}
x(0) \\
Y_{1,0} \\
Y_{2,0}
\end{array}\right),
$$

where $Y_{1,0}$ and $Y_{1, T}$ are defined in equations 38 and 41, and

$$
\begin{aligned}
Y_{2,0} & :=\int_{t_{\mathrm{w}}}^{1} \int_{t_{\mathrm{w}}-1}^{s_{1}-1} \mathrm{e}^{-a s_{2}} x_{0}\left(s_{2}\right) \mathrm{d} s_{2} \mathrm{~d} s_{1}, \\
Y_{2, T} & :=\int_{t_{\mathrm{w}}}^{1} \int_{t_{\mathrm{w}}-1}^{s_{1}-1} \mathrm{e}^{-a s_{2}} x_{T}\left(s_{2}\right) \mathrm{d} s_{2} \mathrm{~d} s_{1} .
\end{aligned}
$$

Here, $\Psi_{2}$ is a $3 \times 3$ transition matrix (not presented here in detail).

It can be shown that, for the general case $n+1-t_{\mathrm{w}}<T \leq n+t_{\mathrm{w}}$, the system can always be described by an $(n+1) \times(n+1)$ transition matrix, denoted by $\Psi_{n}$. In this case, deadbeat control cannot be achieved, since $(n+1)$ poles cannot, in general, be placed at the origin using only one control parameter, $d$.

\subsubsection{Case $t_{\mathrm{w}}<1$, with $n<T \leq n+1-t_{\mathrm{w}}$}

Consider first the case $n=1$, i.e. $1<T \leq 2-t_{\mathrm{w}}$ (see Figure 7b). The solution after one act-and-wait period can be determined according to equation 40 , but the weighted integral $Y_{1, T}$ is now given by

$$
Y_{1, T}=\int_{t_{\mathrm{w}}-1}^{1-T} \mathrm{e}^{-a s} x_{\mathrm{p} 2}(s+T) \mathrm{d} s+\int_{1-T}^{0} \mathrm{e}^{-a s} x_{\mathrm{p} 3}(s+T) \mathrm{d} s
$$

instead of equation 43, since now $t_{\mathrm{w}}-1<1-T$. From equation 52, it can be seen that $Y_{1, T}$ can only be determined if the initial function $x_{0}(s)$ is known over $s \in\left[t_{\mathrm{w}}-1,1-T\right]$. Thus, in this case, no finite-dimensional discrete map can be constructed in a similar way to that in Section 4.2.1. Similarly, it can be shown that, for any $n=2,3, \ldots$, the algorithm 
of constructing a finite-dimensional discrete map does not work. Note, however, that the fact that a finite-dimensional map cannot be constructed in this way does not assure that the system is infinite-dimensional.

\subsubsection{Case $t_{\mathrm{w}}<1$, with $n-1+t_{\mathrm{w}}<T \leq n$}

Consider first the case $n=1$, i.e. $t_{\mathrm{w}}<T \leq 1$ (see Figure 7c). The state after one act-andwait period is given by

$$
x(T)=\mathrm{e}^{a T} x(0)-d \underbrace{\int_{t_{\mathrm{w}}-1}^{T-1} \mathrm{e}^{a(T-s-1)} x_{0}(s) \mathrm{d} s}_{=: V_{1,0}} .
$$

This is a linear combination of $x(0)$ and the weighted integral $V_{1,0}$. In order to obtain a discrete map, the value of

$$
V_{1, T}:=\int_{t_{\mathrm{w}}-1}^{T-1} \mathrm{e}^{a(T-s-1)} x_{T}(s) \mathrm{d} s
$$

should also be expressed as a linear combination of $x(0)$ and $V_{1,0}$. Here, $x_{T}$ is given by

$$
x_{T}(s)=\left\{\begin{array}{ccc}
x_{0}(s+T), & \text { if } \quad-1<s \leq-T, \\
x_{\mathrm{p} 1}(s+T), & \text { if } \quad-T<s \leq t_{\mathrm{w}}-T, \\
x_{\mathrm{p} 2}(s+T), & \text { if } \quad t_{\mathrm{w}}-T<s \leq 0 .
\end{array}\right.
$$

where the functions $x_{\mathrm{p} 1}(s)$ and $x_{\mathrm{p} 1}(s)$ are defined in equations 36 and 37 , respectively. Thus,

$$
V_{1, T}=\int_{t_{\mathrm{w}}-1}^{t_{\mathrm{w}}-T} \mathrm{e}^{a(T-s-1)} x_{\mathrm{p} 1}(s+T) \mathrm{d} s+\int_{t_{\mathrm{w}}-T}^{T-1} \mathrm{e}^{a(T-s-1)} x_{\mathrm{p} 2}(s+T) \mathrm{d} s .
$$

It can be seen that $V_{1, T}$ can only be determined if the initial function $x_{0}(s)$ is known over $s \in\left[t_{\mathrm{w}}-1, T-1\right]$. Thus, the algorithm of constructing a finite-dimensional discrete map does not work in this case either.

One can say that the system with $n-1+t_{\mathrm{w}}<T<n+1-t_{\mathrm{w}}$ can generally be described by the monodromy operator $\mathcal{U}(\mathcal{T})$, which, in general (but not necessary), has infinitely many poles.

\subsection{Number of poles for arbitrary $t_{\mathrm{w}}$}

Based on the previous subsections, the number of poles of equation 12 is summarized in Table 1. The geometric representation of the different cases in the plane $\left(t_{\mathrm{a}}, t_{\mathrm{w}}\right)$ are presented in Figure 8. Question marks refers to the fact that the system is not necessarily infinitedimensional if the algorithm for constructing a finite dimensional discrete map fails, as it was shown in Sections 4.2.2 and 4.2.3. 
Table 1. Summary of the dimension of the monodromy operator of equation 12.

\begin{tabular}{ccc}
\hline Condition for $t_{\mathrm{w}}$ and $t_{\mathrm{a}}$ & Monodromy operator & Number of nonzero poles \\
\hline$t_{\mathrm{w}} \geq 1$ & $\left(\begin{array}{cc}\Phi_{n} & O \\
f_{n} & \mathcal{O}\end{array}\right)$ & 1 \\
$n-1<t_{\mathrm{a}} \leq n$ & $\left(\begin{array}{cc}\Psi_{n} & O \\
f_{x, Y} & \mathcal{O}\end{array}\right)$ & \\
$t_{\mathrm{w}}<1$ & & \\
$n-1<t_{\mathrm{a}} \leq n$ & & \\
$t_{\mathrm{w}} \geq \frac{n+1}{2}-\frac{t_{\mathrm{a}}}{2}$ & $\mathcal{U}(T)$ & $\infty(?)$ \\
$t_{\mathrm{w}}<1$ & & \\
$n-1<t_{\mathrm{a}} \leq n$ & & \\
$t_{\mathrm{w}}<\frac{n+1}{2}-\frac{t_{\mathrm{a}}}{2}$ & & \\
\hline
\end{tabular}

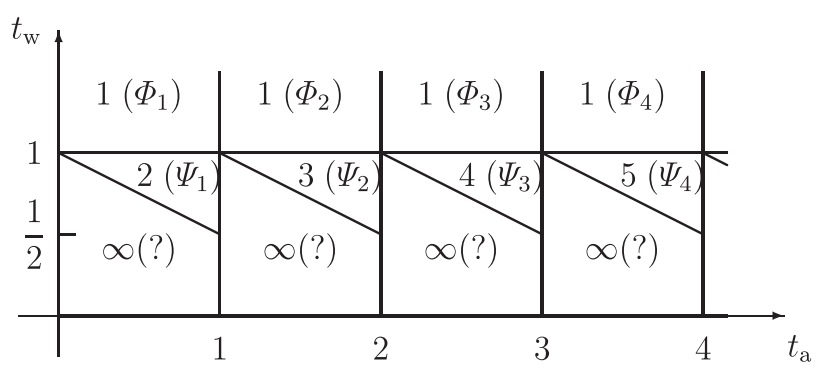

Figure 8. Chart of the dimension of the monodromy operator of equation 12.

\section{OPTIMIZATION OF OTHER PARAMETERS BASED ON PERFOR- MANCE}

The system performance is characterized by the index

$$
J=\frac{1}{2} Q J_{1}+\frac{1}{2} R J_{2}+\frac{1}{2} W J_{3}
$$

where

$$
J_{1}=\int_{0}^{\infty} x^{2}(s) \mathrm{d} s, \quad J_{2}=\int_{0}^{\infty} u^{2}(s) \mathrm{d} s, \quad J_{3}=x_{f}^{2} .
$$

In the above, $J_{1}$ roughly corresponds to the rate of decay of the signal, $J_{2}$ to the control effort, and $J_{3}$ corresponds to the accuracy of the final position for constant actuator disturbances such as Coulomb friction. The constants $Q, R$ and $W$ are weights. The aim is to find the control parameters that minimize the performance index $J$. Since the weights $Q, R$ and $W$ might be different for different problems, we will investigate the terms $J_{1}, J_{2}$ and $J_{3}$ individually for the deadbeat case $t_{\mathrm{w}}>1,0<t_{\mathrm{a}} \leq 1$ when $t_{\mathrm{a}}=\mathrm{e}^{a} / d$. For this case, the control input and the solution can be given in the piecewise form as (see Figure 9): 

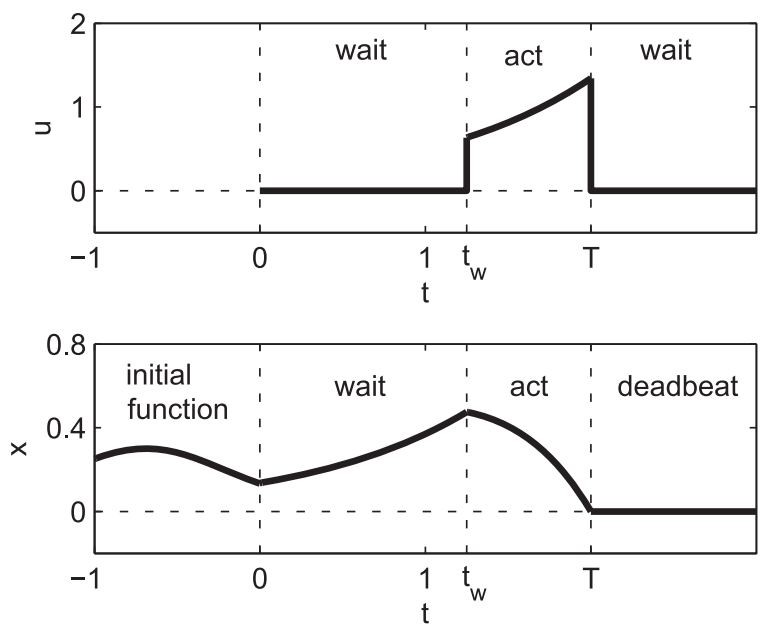

Figure 9. The input and the response of the system in the deadbeat case with $a=1$ and $t_{\mathrm{a}} \leq 1$.

$$
\begin{aligned}
& u(t)=\left\{\begin{array}{ccc}
0, & \text { if } \quad 0<t \leq t_{\mathrm{w}}, \\
d \mathrm{e}^{a(t-1)} x(0), & \text { if } t_{\mathrm{w}}<t \leq t_{\mathrm{w}}+t_{\mathrm{a}}, \\
0, & \text { if } \quad t_{\mathrm{w}}+t_{\mathrm{a}}<t,
\end{array}\right. \\
& x(t)=\left\{\begin{array}{ccc}
\mathrm{e}^{a t} x(0), & \text { if } & 0<t \leq t_{\mathrm{w}}, \\
d \mathrm{e}^{a t}\left(1-\mathrm{e}^{-a}\right)\left(t-t_{\mathrm{w}}\right) x(0), & \text { if } t_{\mathrm{w}}<t \leq t_{\mathrm{w}}+t_{\mathrm{a}}, \\
0, & \text { if } \quad t_{\mathrm{w}}+t_{\mathrm{a}}<t .
\end{array}\right.
\end{aligned}
$$

Evaluation of $J_{1}$ using equation 60 and substitution of $t_{\mathrm{a}}=\mathrm{e}^{a} / d$ gives

$$
J_{1}=\left(\frac{1}{2 a}\left(\mathrm{e}^{2 a t_{\mathrm{w}}}-1\right)+\frac{\left(\mathrm{e}^{\frac{2 a e^{a}}{d}}-1\right) d^{2}-2 d a \mathrm{e}^{a}-2 a^{2} \mathrm{e}^{2 a}}{4 a^{3}} \mathrm{e}^{2 a\left(t_{\mathrm{w}}-1\right)}\right) x^{2}(0)
$$

From here, it can be seen that

$$
\frac{\partial J_{1}}{\partial d}<0
$$

i.e. the larger the control gain $d$, the less the $J_{1}$. Thus, the optimal gain with respect to $J_{1}$ is $d_{\mathrm{J} 1 \mathrm{opt}} \rightarrow \infty$, with the corresponding acting period length $t_{\mathrm{a} \mathrm{J} 1 \mathrm{opt}} \rightarrow 0$.

Evaluation of term $J_{2}$ using euqation 59 with $t_{\mathrm{a}}=\mathrm{e}^{a} / d$ gives

$$
J_{2}=\frac{\left(\mathrm{e}^{\frac{2 a e^{a}}{d}}-1\right) \mathrm{e}^{2 a\left(t_{\mathrm{w}}-1\right)}}{2 a} d^{2} x^{2}(0) \text {. }
$$



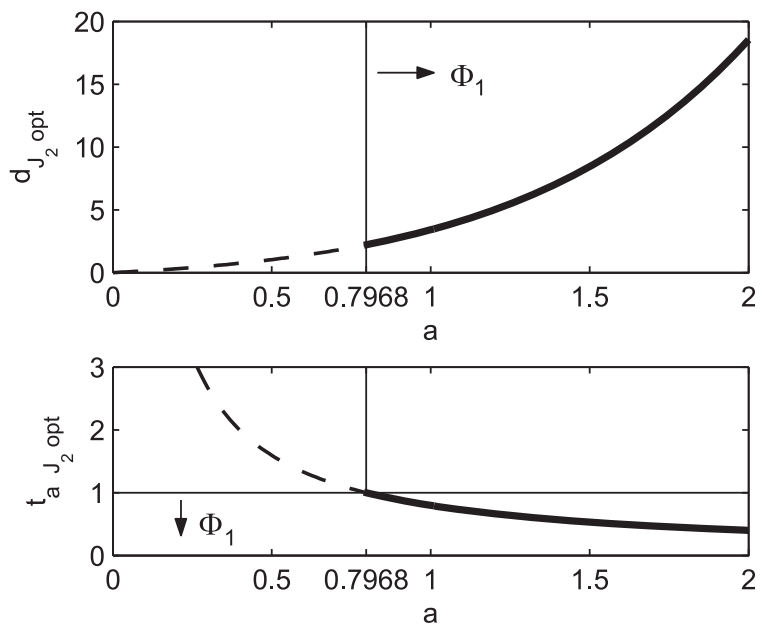

Figure 10. Optimal gain $d$ and acting period length $t_{\mathrm{a}}$ for deadbeat control with $t_{\mathrm{a}}<1$.

Partial derivation with respect to $d$ gives the control gain where $J_{2}$ is minimal,

$$
d_{\mathrm{J} 2 \mathrm{opt}}=\frac{2 a \mathrm{e}^{a}}{W_{\mathrm{L}}\left(-2 \mathrm{e}^{-2}\right)+2} .
$$

Here $W_{\mathrm{L}}(s)$ is the so-called Lambert function, defined as $W_{\mathrm{L}}(s) \mathrm{e}^{W_{\mathrm{L}}(s)}=s$. Lambert functions often arise in computational techniques for DDEs (see, for example, Asl and Ulsoy, 2003). The corresponding optimal acting period length is

$$
t_{\mathrm{a} \mathrm{J} 2 \mathrm{opt}}=\frac{\mathrm{e}^{a}}{d_{\mathrm{J} 2 \mathrm{opt}}}=\frac{W_{\mathrm{L}}\left(-2 \mathrm{e}^{-2}\right)+2}{2 a} .
$$

Since the current analysis is restricted to the case $t_{\mathrm{a}} \leq 1$, these optimal values are available only if $a \geq 1+W_{\mathrm{L}}\left(-2 \mathrm{e}^{-2}\right) / 2=0.7968$. Here, $d_{\mathrm{J} 2 \mathrm{opt}}=2.2184$. The corresponding optimal parameters are presented in Figure 10 by solid lines. Optimal parameters for the cases when $t_{\mathrm{a}}>1$ can be obtained similarly.

The term $J_{3}$ depends only on the final position given by equation 7 ,

$$
J_{3}=x_{f}^{2}=\frac{\Delta^{2}}{(d-a)^{2}} \text {. }
$$

Since $J_{3}$ is inversely proportional to the square of gain $d$, it decreases with an increase in the control gain. Hence, the optimal gain considering $J_{3}$ is given by $d_{\mathrm{J} 3 \text { opt }} \rightarrow \infty$ similar to the one corresponding to $J_{1}$.

Note that the minimum of $J_{1}$ and $J_{3}$ are obtained at $d \rightarrow \infty$, while the minimum of $J_{2}$ (given by equation 64) is obtained at finite values of the control gain $d$ with a corresponding acting time $t_{\mathrm{a}}$, both depending on the system parameter $a$, as shown in Figure 10. Depending 
on the problem at hand, the weights $Q, R$ and $W$ can be chosen and an overall optimal value can be determined by minimizing the composite performance index $J$ given by equation 57 . This minimization process might be subject to problem-specific physical constraints such as limitations on the maximum control force or the maximum acting period.

\section{ROBUSTNESS OF DEADBEAT CONTROL}

In the case of deadbeat control, all the poles are located at the origin. However, small perturbations in the system or control parameters destroy this property, and larger perturbations may even destabilize the originally deadbeat system. According to Michiels and Roose (2003), the stability radius can be used as a measure of the robustness of stability. The stability radius corresponds to the smallest perturbations that result in a shift of a pole outside the closed unit disc of the complex plane and, hence, cause instability. For given parameters $a$, $d, t_{\mathrm{w}}$ and $t_{\mathrm{a}}$, denote the spectral radius of the monodromy operator $\mathcal{U}(T)$ by $\sigma_{1}\left(a, d, t_{\mathrm{w}}, t_{\mathrm{a}}\right)$. For instance, the stability radius of the deadbeat control system with respect to changes in $a$ can be defined as

$$
r_{a}=\inf _{\Delta a \in \mathbb{R}}\left\{|\Delta a / a|: \sigma_{1}\left(a+\Delta a, d_{\mathrm{db}}, t_{\mathrm{w}}, t_{\mathrm{a}}\right) \geq 1\right\}
$$

where $d_{\mathrm{db}}$ is the control gain that results in deadbeat control. For fixed $a$ and $t_{\mathrm{a}}$, the gain $d_{\mathrm{db}}$ can be given by solving equation 32 for $d$. We can similarly define stability radii $r_{d}, r_{t_{\mathrm{w}}}$ and $r_{t_{\mathrm{a}}}$.

In the case $t_{\mathrm{w}} \geq 1$ and $0<t_{\mathrm{a}} \leq 1$, the stability radius $r_{d}$ can be determined in closed form:

$$
\left.\begin{array}{c}
\Phi_{1}=0 \Rightarrow d_{\mathrm{db}}=\frac{\mathrm{e}^{a}}{t_{\mathrm{a}}} \\
\Phi_{1}= \pm 1 \Rightarrow d_{ \pm 1}=\frac{\mathrm{e}^{a} \mp \mathrm{e}^{a\left(1-t_{\mathrm{a}}-t_{\mathrm{w}}\right)}}{t_{\mathrm{a}}}
\end{array}\right\} \Rightarrow r_{d}=\mathrm{e}^{-a\left(t_{\mathrm{a}}+t_{\mathrm{w}}\right)}=\mathrm{e}^{-a T}
$$

Note that the deadbeat parameter points are right in the middle of the domain of stability. This means that the deadbeat parameter points are just those where the robustness of stability is the largest in the sense of Michiels and Roose (2003). It can clearly be seen that $r_{d}$ decreases with increasing $a, t_{\mathrm{a}}$ and $t_{\mathrm{w}}$.

As opposed to $r_{d}$, stability radii $r_{a}, r_{t_{\mathrm{w}}}$ and $r_{t_{\mathrm{a}}}$ cannot be expressed in closed form, but they can be determined numerically. For given parameters $a$ and $t_{\mathrm{a}}$, the deadbeat control gain $d_{\mathrm{db}}$ can be determined using equation 32, and the stability of the system can be followed numerically for varying $a, t_{\mathrm{a}}$ and $t_{\mathrm{w}}$. The minimum absolute value of $\Delta a, \Delta t_{\mathrm{a}}$ and $\Delta t_{\mathrm{w}}$, which result in an unstable system gives $r_{a}, r_{t_{\mathrm{a}}}$ and $r_{t_{\mathrm{w}}}$.

For fixed $n-1<t_{\mathrm{a}} \leq n$ and $t_{\mathrm{w}} \geq 1$, stability radius $r_{a}$ can be determined numerically using equation 25 for $\Phi_{n}$. However, during the numerical computation of $r_{t_{\mathrm{a}}}$, the condition $n-1<t_{\mathrm{a}}+\Delta t_{\mathrm{a}} \leq n$ should be monitored in order to use the proper value of $n$ for $\Phi_{n}$.

It can be seen that stability radius $r_{t_{\mathrm{w}}}$ always satisfies 

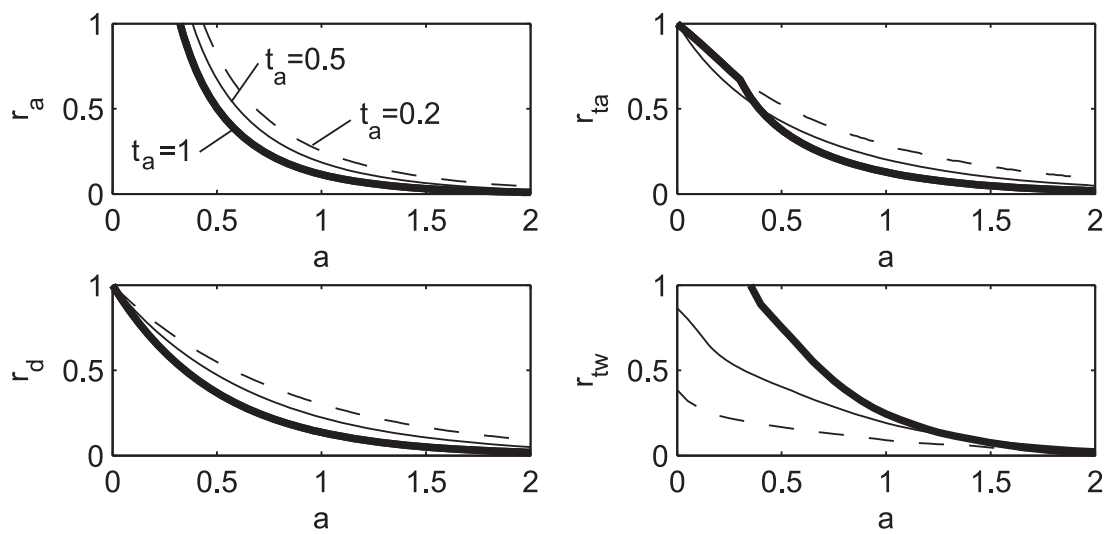

Figure 11. Robustness of deadbeat control for $t_{\mathrm{w}}=1$. Thick lines: $t_{\mathrm{a}}=1$. Thin lines: $t_{\mathrm{a}}=0.5$. Dashed lines: $t_{\mathrm{a}}=0.2$.

$$
r_{t_{\mathrm{w}}}>1-1 / t_{\mathrm{w}}
$$

This is due to the fact that if $t_{\mathrm{w}}+\Delta t_{\mathrm{w}}>1$, i.e. $t_{\mathrm{w}}$ is large enough so that it never gets smaller than 1 for any disturbances, then the system is described by $\Phi_{n}$, and the corresponding deadbeat condition 32 does not depend on $t_{\mathrm{w}}$. The only possible way to lose stability is to perturb $t_{\mathrm{w}}$ by $\Delta t_{\mathrm{w}}<1-t_{\mathrm{w}}$ in order to get $t_{\mathrm{w}}+\Delta t_{\mathrm{w}}<1$, which corresponds to equation 69. In this case, equation 25 for $\Phi_{n}$ is not valid any more, since the dimension of the system changes according to Figure 8 , and the system may also become infinite-dimensional. Then, $r_{t_{\mathrm{w}}}$ should be determined using other numerical techniques. Here, the semi-discretization method was used.

Figure 11 shows the stability radii of the deadbeat control as a function of system parameter $a$ for different acting period length $t_{\mathrm{a}}$, while the waiting period length is fixed to $t_{\mathrm{w}}=1$. It can be seen that stability radii $r_{a}, r_{d}$ and, for $a>0.5, r_{t_{\mathrm{a}}}$ increase with decreasing $t_{\mathrm{a}}$, while the stability radius $r_{t_{\mathrm{w}}}$ increases with increasing $t_{\mathrm{a}}$.

Figure 12 shows the stability radii of the deadbeat control for different waiting period length $t_{\mathrm{w}}$, while the acting period length is fixed to $t_{\mathrm{a}}=1$. It can be seen that stability radii $r_{a}$, $r_{d}$ and $r_{t_{\mathrm{a}}}$ decrease with increasing $t_{\mathrm{w}}$, while the stability radius $r_{t_{\mathrm{w}}}$ increases with increasing $t_{\mathrm{w}}$. Note that equation 69 gives here $r_{t_{\mathrm{w}}}>1 / 2$ for $t_{\mathrm{w}}=2$ and $r_{t_{\mathrm{w}}}>4 / 5$ for $t_{\mathrm{w}}=5$, as can also be seen in Figure 12.

Stability radii $r_{t_{\mathrm{w}}}$ in Figures 11 and 12 shows that the system does not get suddenly unstable for perturbations in $t_{\mathrm{w}}$, even in the case $t_{\mathrm{w}}+\Delta t_{\mathrm{w}}<1$ when the system may also become infinite-dimensional. If $t_{\mathrm{w}}=2$ or 5 , then the system is more robust against disturbances in $t_{\mathrm{w}}$ than against disturbances in $a, d$ and $t_{\mathrm{a}}$. For $t_{\mathrm{w}}=1$ and $t_{\mathrm{a}}=1, r_{t_{\mathrm{w}}}$ have similar characteristics to the stability radii $r_{a}, r_{d}$ or $r_{t_{\mathrm{a}}}$. However, if $t_{\mathrm{w}}=1$ and $t_{\mathrm{a}}=0.5$ or 0.2 , then the robustness against $t_{\mathrm{w}}$ is worse than that against the other parameters. Figures 11 and 12 also show that all the four stability radii $r_{a}, r_{d}, r_{t_{\mathrm{a}}}$ and $r_{t_{\mathrm{w}}}$ decrease with increasing system parameter $a$.

Robustness against disturbances in the time delay can be characterized by the stability radius with respect to changes in the delay. Due to the scaling of the time delay to $\tau=1$, 

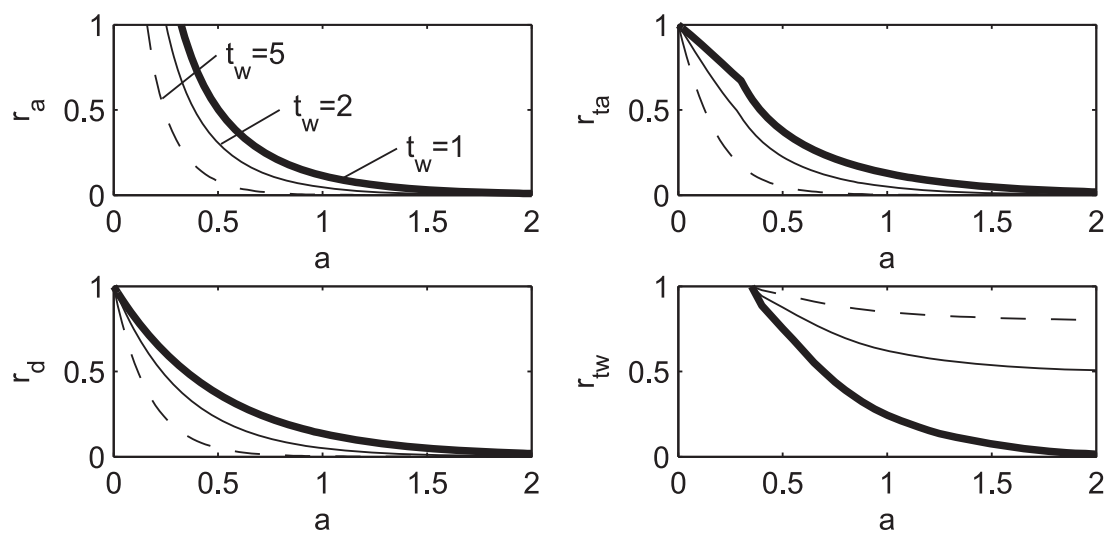

Figure 12. Robustness of deadbeat control for $t_{\mathrm{a}}=1$. Thick lines: $t_{\mathrm{w}}=1$. Thin lines: $t_{\mathrm{w}}=2$. Dashed lines: $t_{\mathrm{w}}=5$.

disturbances in the delay parameter are transformed to disturbances in the parameters $a, d$, $t_{\mathrm{a}}$ and $t_{\mathrm{w}}$. If the time delay is changed from 1 to $1+\Delta \tau$, then rescaling of the time gives $\tilde{a}=a(1+\Delta \tau), \tilde{d}=d(1+\Delta \tau), \tilde{t}_{\mathrm{a}}=t_{\mathrm{a}} /(1+\Delta \tau)$ and $\tilde{t}_{\mathrm{a}}=t_{\mathrm{w}} /(1+\Delta \tau)$, where tilde denotes the parameters in the rescaled time. Thus the robustness of the system against disturbances in the time delay is a combination of the robustness against disturbances in $a, d, t_{\mathrm{a}}$ and $t_{\mathrm{w}}$. The corresponding stability radius can be defined as

$$
r_{\tau}=\inf _{\Delta \tau \in \mathbb{R}}\left\{|\Delta \tau| / 1: \sigma_{1}\left(a(1+\Delta \tau), d_{\mathrm{db}}(1+\Delta \tau), t_{\mathrm{w}} /(1+\Delta \tau), t_{\mathrm{a}} /(1+\Delta \tau)\right) \geq 1\right\}
$$

If $t_{\mathrm{w}} /(1+\Delta \tau) \geq 1$ and $0<t_{\mathrm{a}} /(1+\Delta \tau) \leq 1$, then this transformation does not affect the terms $a\left(t_{\mathrm{a}}+t_{\mathrm{w}}\right)$ and $d t_{\mathrm{a}}$ of $\Phi_{1}$ in equation 30 , and the only term that changes is $\mathrm{e}^{-a}$. In this case, substitution of the deadbeat condition $d_{\mathrm{db}}=\mathrm{e}^{a} / t_{\mathrm{a}}$ into $\Phi_{1}= \pm 1$ gives the stability radius $r_{\tau}$ as

$$
r_{\tau}=\frac{1}{a} \ln \left(1+\mathrm{e}^{-a\left(t_{\mathrm{a}}+t_{\mathrm{w}}\right)}\right) .
$$

The case $n-1<t_{\mathrm{a}} /(1+\Delta \tau) \leq n$ can be investigated similarly, but $\Phi_{n}$ should be used instead of $\Phi_{1}$. If the perturbation yields $t_{\mathrm{w}} /(1+\Delta \tau)<1$ then the system's dimension changes according to Figure 8 and $\Phi_{n}$ cannot be used, but stability can be determined using numerical techniques, such as the semi-discretization method.

Figure 13 shows the stability radius $r_{\tau}$ of the deadbeat control as a function of system parameter $a$ for different act-and-wait period lengths. It can be seen that the stability radius increases with decreasing $t_{\mathrm{w}}$ and with increasing $t_{\mathrm{a}}$. The point is that, even if $t_{\mathrm{w}}$ is chosen to be close to 1 , and disturbances in the parameters results in $t_{\mathrm{w}}<\tau=1$, the stability of the system does not change radically. Moreover, the robustness against disturbance in the delay $\tau$ or the waiting period length $t_{\mathrm{w}}$ show similar characteristic as the robustness against the other parameters $a, d$ and $t_{\mathrm{a}}$. 

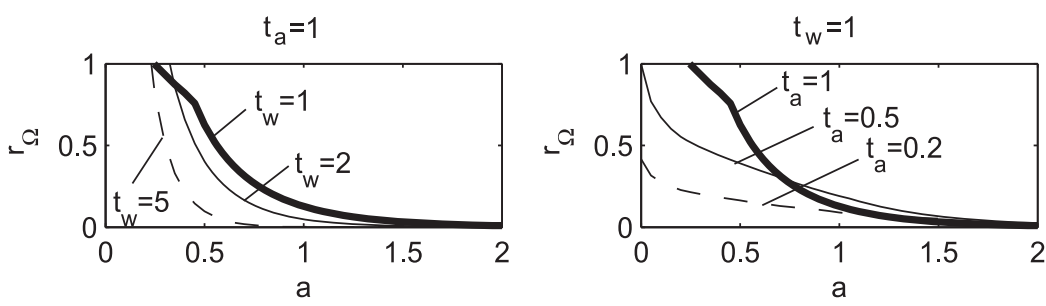

Figure 13. Robustness of deadbeat control against disturbances in the time delay, with the nominal value $\tau=1$.

\section{CONCLUSION}

The act-and-wait concept was investigated for the delayed first-order lag compensator in equation 12 with respect to the state parameter $a$ and the three control parameters, namely, the control gain $d$, the length $t_{\mathrm{a}}$ of the acting period and the length $t_{\mathrm{w}}$ of the waiting period. The delay parameter $\tau$ was scaled to 1 . It was shown that if $t_{\mathrm{w}} \geq 1$, then the system can be transformed into a scalar discrete map, and deadbeat control can always be achieved for any system parameter $a$. If $t_{\mathrm{w}}$ and $t_{\mathrm{a}}$ are fixed so that $t_{\mathrm{w}}>1$ and $t_{\mathrm{a}}>1$, then more values of $d$ can be found that result in deadbeat control. In these cases, there are some parameter domains (gray region in Figure 5) that are bounded by deadbeat parameter curves. In these domains, both increase and decrease of the control gain $d$ push the system to the direction of deadbeat. However, if the control gain $d$ is limited so that it cannot be larger than $\mathrm{e}^{a-1}$, then deadbeat control cannot be attained.

It was shown that, for some combinations of the act-and-wait period lengths, the system can still be transformed to a finite-dimensional discrete map if $1 / 2 \leq t_{\mathrm{w}}<1$. Numerical stability charts provided evidence that the transition between the cases $t_{\mathrm{w}}<1$ and $t_{\mathrm{w}}>1$ is smooth, i.e. there are no abrupt changes in the stability properties if the waiting period length or the time delay is perturbed, although, deadbeat disappears for $t_{\mathrm{w}}<1$.

In the case of deadbeat control, the system performance was analyzed with respect to three different terms of the performance index, the fastest settling signal $J_{1}$, the minimal control effort $J_{2}$ and the minimal error for constant actuator disturbances $J_{3}$. It was shown that the minimum of $J_{1}$ and $J_{3}$ is obtained if $d \rightarrow \infty$ with $t_{\mathrm{a}} \rightarrow 0$, while the minimum of $J_{2}$ can be found at certain finite values of $d$ and $t_{\mathrm{a}}$ (shown in Figure 10) depending on the system parameter $a$.

The robustness of the deadbeat control strategy with respect to perturbations in the system and control parameters was also analyzed using stability radii as a measure. It was found that the stability radius of deadbeat control is sufficiently large if the system parameter $a$ and the acting period length $t_{\mathrm{a}}$ are small and the waiting period length is $t_{\mathrm{w}}=1$, i.e. the waiting period is equal to the time delay. However, the stability radii decrease with an increase in the system parameter.

Acknowledgements. The authors acknowledge the support from the Centre de Recerca Matemàtica (CRM), Barcelona, Thematic Research Term on Non-Smooth Complex Systems. Tamas Insperger was supported by the János Bolyai Research Scholarship of the Hungarian Academy of Sciences and the Hungarian National Science Foundation under grant no. OTKA K72911. Gábor Stépán was supported by the Hungarian National Science Foundation under grant no. OTKA T068910. 


\section{REFERENCES}

Aeyels, D. and Willems, J. L., 1992, "Pole assignment for linear time-invariant systems by periodic memoryless output feedback," Automatica 28(6), 1159-1168.

Allwright, J. C., Astolfi, A., and Wong, H. P., 2005, “A note on asymptotic stabilization of linear systems by periodic, piece-wise constant output feedback," Automatica 41(2), 339-344.

Artstein, Z. and Weiss, G., 2005, "State nullification by memoryless output feedback," Mathematics of Control, Signals, and Systems 17, 38-56.

Asl, F. M. and Ulsoy, A. G., 2003, "Analysis of a system of linear delay differential equations," Journal of Dynamic Systems, Measurement, and Control 125, 215-223.

Bhatt, S. J. and Hsu, C. S., 1966, "Stability criteria for second-order dynamical systems with time lag," Journal of Applied Mechanics 33E(1), 113-118.

Boikov, I. V., 2005, "The Brockett stabilization problem," Automation and Remote Control 66(5), 746-751.

Breda, D., Maset, S., and Vermiglio, R., 2004, "Computing the characteristic roots for delay differential equations," IMA Journal of Numerical Analysis 24, 1-19.

Brockett, R. W., 1998, “A stabilization problem,” in Open Problems in Mathematical Systems and Control Theory, Communications and Control Engineering, Blondel, V. D., Sontag, E. D., Vidyasagar, M., and Willems, J. C. (eds), Springer, Berlin, pp. 75-78 (chapter 16).

Budak, E. and Altintas Y., 1998, "Analytical prediction of chatter stability in milling - part I: general formulation," Journal of Dynamic Systems, Measurement, and Control 120, 22-30.

Butcher, E. A., Ma, H., Bueler, E., Averina, V., and Szabó, Zs., 2004, “Stability of time-periodic delay-differential equations via Chebyshev polynomials," International Journal for Numerical Methods in Engineering 59(7), 895-922.

Cushing, J. M., 1977, "Integrodifferential equations and delay models in population dynamics," in Lecture Notes in Biomathematics 20, Springer, Berlin, Heidelberg, New York.

Diekmann, O., van Gils, S. A., Lunel, S. M. V., and Walther, H.-O., 1995, Delay Equations, Springer, New York.

Farkas, M., 1994, Periodic Motions, Springer-Verlag, New York.

Floquet, M. G., 1883, “Équations différentielles linéaires a coefficients péridiques," Annales Scientifiques de lÉcole Normale Supérieure 12, 47-89.

Hale, J. K. and Lunel, S. M. V., 1993, Introduction to Functional Differential Equations, Springer-Verlag, New York.

Hayes, N. D., 1950, "Roots of the transcendental equations associated with a certain differential-difference equations," Journal of London Mathematical Society 25, 226-232.

Insperger, T., 2006, "Act and wait concept for time-continuous control systems with feedback delay," IEEE Transactions on Control Systems Technology 14(5), 974-977.

Insperger, T. and Stépán, G., 2002a, "Stability chart for the delayed Mathieu equation," Proceedings of the Royal Society London A 458, 1989-1998.

Insperger, T. and Stépán, G., 2002b, "Semi-discretization method for delayed systems," International Journal for Numerical Methods in Engineering 55(5), 503-518.

Insperger, T. and Stépán, G., 2004, "Stability analysis of turning with periodic spindle speed modulation via semidiscretization," Journal of Vibration and Control 10(12), 1835-1855.

Insperger, T. and Stépán, G., 2007, “Act-and-wait control concept for discrete-time systems with feedback delay,” IET Control Theory and Applications 1(3), 553-557.

Kalmár-Nagy, T., 2005, “A new look at the stability analysis of delayed differential equations," in Proceedings of ASME International Design Engineering Technical Conferences, Long Beach, CA, September 24-28, paper no. DETC2005-84740 (CD-ROM).

Kim, W. S. and Bejczy, A. K., 1993, "Demonstration of a high-fidelity predictive preview display technique for telerobotic servicing in space," IEEE Transactions on Robotics and Automation 9(5), 698-704.

Kolmanovskii, V. B. and Nosov, V. R., 1986, Stability of Functional Differential Equations, Academic Press, London.

Kovács, L. L., Insperger, T. and Stépán, G., 2004, "Teaching-in force control of industrial robots used in medical applications," in Proceedings of 15th CISM-IFToMM Symposium on Robot Design, Dynamics and Control, Montreal, Canada, April 1-4, CD-ROM Rom-04-46.

Leonov, G. A., 2002a, "The Brockett problem for linear discrete control systems," Automation and Remote Control 63(5), 777-781.

Leonov, G. A., 2002b, "Brockett's problem in the theory of stability of linear differential equations," St. Petersburg Mathematical Journal 13(4), 613-628. 
Liu, L. and Kalmar-Nagy, T., 2010, "High dimensional harmonic balance analysis for second-order delay-differential equations," Journal of Vibration and Control (in press).

Mann, B. P. and Patel, B. R., 2010, "Stability of delay equations written as state space models," Journal of Vibration and Control (in press).

Mathieu, E., 1868, "Mémoire sur le mouvement vibratoire dune membrane de forme elliptique," Journal De Mathematiques Pures Et Appliquees 13, 137-203.

Michiels, W., Engelborghs, K., Vansevenant, P., and Roose, D., 2002, “Continuous pole placement for delay equations," Automatica 38(5), 747-761.

Michiels, W. and Roose, D., 2003, “An eigenvalue based approach for the robust stabilization of linear time-delay systems," International Journal of Control 76(7), 678-686.

Moreau, L. and Aeyels, D., 2004, "Periodic output feedback stabilization of single-input single-output continuoustime systems with odd relative degree," Systems and Control Letters 51, 395-406.

Namachchivaya, N. S. and Beddini, R., 2003, "Spindle speed variation for the suppression of regenerative chatter," Journal of Nonlinear Science 13(3), 265-288.

Niculescu, S.-I., 2000, Delay effects on stability: A robust control approach, New York, Springer.

Olgac, N. and Sipahi, R., 2002, "An exact method for the stability analysis of time-delayed linear time-invariant (LTI) systems," IEEE Transactions on Automatic Control 47(5), 793-977.

Shakkottai S., Srikant R. and Meyn S., 2003, "Bounds on the throughput of congestion controllers in the presence of feedback delay," IEEE/ACM Transactions on Networking 12, 972-981.

Stépán, G., 1989, Retarded Dynamical Systems, Longman, Harlow.

Stépán, G. and Insperger, T., 2006, "Stability of time-periodic and delayed systems - a route to act-and-wait control," Annual Reviews in Control 30, 159-168.

Stephenson, A., 1908, “On a new type of dynamical stability," Memoirs and Proceedings of the Manchester Literary and Philosophical Society 52, 1-10.

van der Pol, F. and Strutt, M. J. O., 1928,“On the stability of the solutions of Mathieu's equation,” Philosophical Magazine, and Journal of Science 5, 18-38.

Weiss, G., 2005, "Memoryless output feedback nullification and canonical forms, for time varying systems," International Journal of Control 78(15), 1174-1181. 